\title{
Auditory processing disorders (APD) 30 years of
} experience

\begin{abstract}
ASHA (2005) defined auditory processing disorder (APD) as difficulties in the perceptual processing of auditory information in the central nervous system and the neurobiological activity that underlies that processing and gives rise to the electrophysiological auditory potentials. APD has a negative impact on communication skills, language development, and learning. APD practice is highly challenging, as it requires development of specially designed diagnostic test material and adequate auditory training intervention programs with good theoretical background and collaborating multi-disciplinary team. This review article is focusing on APD practice in Egypt during the last 30years. It started by development of Arabic protocols for diagnosis of APD in adults, followed by development of Arabic test material for children. The next step was development of intervention programs based on neuroplasticity of the central nervous system. An Arabic computer-based remediation program was, therefore, developed. When applied on children having APDs, it proved to be effective and showed promising results. Assessment \& management of APD disorders in Egypt have passed through many stages; a long journey where the highly-challenging disorder was nicely brought into focus in clinical practice and research.
\end{abstract}

Keywords: auditory processing disorders, psychophysical behavioral tests, electrophysiological tests
Volume 8 Issue 5 - 2017

\author{
Somia Tawfik, Amani Shalaby \\ ENT Department Ain Shams University, Egypt
}

Correspondence: Somia Tawfik, Audiovesibular Medicine, ENT Department, Ain Shams University Cairo, 30 ElHarony St.\#3, Nasr City, Cairo, Egypt I I 377, Egypt, Tel 2021005073763, Emaildrssomaia@hotmail.com

Received: August 01, 2017 | Published: October 02, 2017
Abbreviations: APD, auditory processing disorders; CAPD, central auditory processing disorders

\section{Introduction}

ASHA (2005) defined auditory processing disorders (APD) as "difficulties in the perceptual processing of auditory information in the central nervous system and the neuro-bio $\neg$ logic activity that underlies that processing and gives rise to the electro-physiologic auditory potentials". ${ }^{1}$ The operational definition of APD is poor performance in one or more of auditory skills. Thus, difficulties in localizing sounds in space, discriminating sounds, recognizing auditory patterns, responding to the temporal aspects of sound (temporal integration, temporal discrimination, temporal ordering, and temporal masking), and extracting auditory information from non-ideal acoustic environments are all examples of APD., ${ }^{2,3}$

ASHA guidelines (2005) stated that diagnosis of APD should be made on the basis of a carefully selected battery of sensitive and specific behavioral tests and electro-physiologic procedures supplemented by observation and detailed case history. The diagnosis should be made by audiologists who have been properly educated and trained in the area of APD, including the administration and interpretation of central tests. Acoustic control of both the test stimuli and the testing environment, and at times special equipment, are all necessary to diagnose APD. Multi-disciplinary assessment complements audiological diagnosis by revealing functional deficits associated with the APD, identifying co-morbid conditions, and informing intervention plans. Likewise, intervention typically requires a multidisciplinary team given the potential impact of APD on listening, communication, academic success, job performance, and social function, as well as the frequent co-morbidity of this disorder with related language, learning, and cognitive disorders.

In 1973, Norma Rees ${ }^{4}$ published a well-known and often quoted article at the time called: "Auditory Processing Factors in Language
Disorders". Dr. Rees went on to contend that: (a) a failure in auditory processing is cited as a major cause of language and learning disorders in children and adults. A long way since 1973, practice of diagnosis and management of subjects with APD has shown remarkable progress. Audiological approaches to the diagnosis, management and rehabilitation of affected persons were summarized by. ${ }^{2-4}$

Nowadays, APD is practiced in many centers all over the world; testing and remediation material being developed in several languages. In Egypt, this practice began 30years ago. It was a challenging task, as it required adequate knowledge of the neurobiology of the central auditory nervous system, developing test material in Arabic language and establishing database for norms. It was mandatory to arrange appropriate set-up for testing with proper training on test administration and test scoring. Thus, the first stage was to develop diagnostic protocols and the second stage was development of intervention plans.

\section{I-Diagnosis of APD}

The era of APD diagnosis in Egypt began by the pioneering work conducted at the Audiology Unit, Faculty of Medicine, Ain Shams University. The first stage was development of specially-designed Arabic test material for assessment of central abilities in adults. In 1984, Soliman et al. ${ }^{5}$ developed the Arabic Staggered Spondaic Word test. This was followed by development of a complete Arabic test battery designed to evaluate different auditory skills in adults by Tawfik et al., ${ }^{6}$ This included low pass filtered speech test, speech in noise test, synthetic sentence identification test and time compressed speech test. In addition, non-verbal tests were included in the battery for assessment of temporal processing abilities. These were pitch pattern sequence test developed by Pinheiro \& Musiek ${ }^{7}$ and auditory fusion test developed and revised by Keith \& McCroskey. ${ }^{8}$

The second stage included set up of equipment for testing, preparing scoring sheets, establishing norms on the tests and check 
for reliability and validity of the developed tests. Then, a clinic for evaluating adult patients with APD was established in 1987 using a battery of behavioral (psychophysical) tests. APD clinic evaluated patients with neuro-psychiatric disorders such as multiple sclerosis, brain tumors, epileptic patients and stroke patients., ${ }^{9,10}$

In 1995, APD practice has expanded to evaluate children by development and standardization of Arabic test material for children by Tawfik et al., ${ }^{11}$ The developed test battery included low pass filtered speech test, speech in noise test, competing sentence test and dichotic digit test. ${ }^{12}$ Tests were standardized and age-specific norms were established.

It is known that evaluation of children requires a team of audiologists, phoniatricians, psychosocial workers and neuropsychiatric physicians in order to reach appropriate diagnosis and differentiate isolated APD from associated or co-morbid conditions such as attention deficit disorder, specific language impairment and autism. Such multidisciplinary team was established in Egypt in 1998. Children who were referred to the clinic had listening, language and/or learning problems. The major cause of referral was learning problems with poor scholastic achievement. Many children with neuro-psychiatric disorders such as attention deficit disorders \& autism were evaluated. ${ }^{13}$ The behavioral psychophysical testing proved to be effective in detecting auditory processing disorders and in identification of the affected auditory abilitie(s).

ASHA Technical Report, ${ }^{1}$ reported that "Electrophysiological and electroacoustic measures are central to the diagnosis of APD, and that auditory evoked potential studies investigating processes from the brainstem to the auditory cortex have increased the understanding of auditory processing. Accordingly, electrophysiological measures were applied to assess children with APD including auditory cognitive potentials (P300) and mismatch negativity by Tawfik \& El-Awady et al., ${ }^{14,15}$ Recently, Nina Kraus and her colleagues indicated that the auditory brainstem response to complex sounds (cABR) provides an objective index of the biological health of the central auditory system and is a sensitive indicator of training-induced neuro-plastic changes. ${ }^{16}$ Taha et al., ${ }^{17}$ studied (cABR) and speech cortical potentials in APD and proved that they are effective tools in APD practice.

\section{Management of APD}

Intervention protocols for Arabic-speaking APD patients were implemented in 2000 using environmental modifications strategy, auditory training programs and compensatory strategies. These strategies were based on studies of neural plasticity as intensive intervention can cause formation of new neural networks by forming new connections between brain cells. Chermak et al., ${ }^{18}$ has reported that stimulation and practice can induce cortical re-organization leading to change in behavior. Accordingly, intensive remediation auditory training programs were developed in Arabic language. These included informal as well as formal programs. Tawfik et al., ${ }^{19}$ developed informal programs in Arabic language with simple material that can be used at home. Formal programs with specially-designed Arabic material to be used in the clinic were developed by Tawfik et al., ${ }^{20,21}$ The first Arabic computer-based auditory training program was released by the Audiology Unit, Ain Shams University in 2006 in collaboration with Horizon Interactive Studios..$^{22,23}$ It included training on affected auditory skills in a game format using graduated approach with re-inforcement. Application of the computer-based auditory training materials in remediation of APD children by Tawfik et al., ${ }^{24}$ showed beneficial improvement during short- and long-term follow up.
Recently, Afifi et al.,25 applied the computer -based remediation program in rehabilitation of children who were fitted with hearing aids and cochlear implants and proved to be a valuable additional rehabilitative tool in this group of children.

\section{Conclusion}

APD practice in Egypt passed through a long road that required knowledge, training and experience in order to help APD patients who needs appropriate diagnosis and intensive intervention. Still, there is a need for further research in order to develop other diagnostic tools such neuro-imaging studies and evaluate extensively the role of neuro-plasticity in APD remediation.

\section{Acknowledgements}

Salah Soliman MD, Professor Emirates of Audiovestibular Medicine, ENT department, Ain Shams University.

\section{Conflicts of interest}

No conflict of interest.

\section{Funding}

None.

\section{References}

1. American Speech-Language-Hearing Association. (Central) auditory processing disorders. Technical Report. 2005

2. American Speech-Language-Hearing Association. Central auditory processing: Current status of research and implications for clinical practice [Technical Report]. American Journal of Audiology. 1996;5(2):41-54.

3. American Academy of Audiology Clinical Practice Guidelines. Guidelines for the Diagnosis, Treatment and Management of Children and Adults with Central Auditory Processing Disorder. Resources/ document library/Documents/CAPD Guidelines. 2010.

4. Rees N. Auditory Processing Factors in Language Disorders: The View from Procrustes Bed. J Speech Hear Disord. 1973;38(3):304-315.

5. Soliman S, Shehata W, Fathallah A. Development of Arabic Staggered Spondaic Word test. Ain Shams medical journal. 1984;4:23-30.

6. Tawfik S, Soliman S, Elkhodry A. Development of Arabic central auditory test battery for adults, unpublished doctor thesis, Audiology unit, ORL Department, Ain Shams University, Egypt. 1986.

7. Pinheiro M, Musiek F. Screening and temporal ordering in the auditory system. In: ML Pinheiro M \& Musiek F (Eds): Assessment of central auditory dysfunction: Foundations and clinical correlates, Baltimore, Williams and Wilkins, Egyp. 1985. p. 291-338.

8. Keith R, McCroskey. Auditory fusion test revisied tt could fill the gap in your CAPD battery. 1999.

9. Tawfik S. Central auditory tests in epileptics. Proceedings of XIV Annual, Ain Shams Medical Congress, Egypt. 1991. p.89-97.

10. Shafik N, Tawfik S, El-Danasoury I, et al. Central Auditory Tests in stroke patients. Unpublished doctor thesis, Audiology unit, ORL Department, Ain Shams University, Egypt. 2012.

11. Tawfik S, Shalaby A. Development and standardization of Arabic Central Test Battery in Children. Proceedings of the XXIII World Congress of the International Association of Logopedics and Phoniatrics, Egypt. 1995. p.25-31. 
12. Weiheba H, Tawfik S, Abdel-Maksoud A. Standardization of two Binaural Dichotic Digits and Dichotic Rhyme Test on Normal Children, Master thesis, Audiology unit, ORL Department, Ain Shams University, Egypt. 2008.

13. Effat S, Tawfik S, Hussein $\mathrm{H}$, et al. Central auditory processing in attention deficit hyperactivity disorder: An Egyptian Study. Middle East Current Psychiatry. 2011;18: 245-252.

14. Tawfik S, El-Danasoury I, Abdel-Maksoud A, et al. Auditory attention and memory tests for children: Development, standardization and application. The Egyptian Journal of otolaryngology. 2002;19(2):31-43.

15. El-Awady M, Tawfik S, El-Danasoury I, et al. Measurement of mismatch negativity in children with sensorineural hearing loss. Unpublished master thesis, Ain Shams University, Egypt. 2006

16. Kraus N, Anderson S. Hearing matters: CABR may improve hearing aid outcomes Hearing Journal. 2012; 65(11):56.

17. Taha T, Hazza N, Elkholy W. Speech-Evoked ABR \& Event Related Potentials: Standardization \& Application in Children with Central Auditory Processing Disorders. Unpublished doctor thesis, Ain Shams University, Egypt. 2011.

18. Chermak GD, Bellis TJ, Musiek FE. Neurobiology, cognitive science and intervention. In: GD Chermak \& FE Musiek (Eds.), Handbook of (central) auditory processing disorder: Comprehensive intervention, Plural Publishing, San Diego, CA, USA. 2007. p.3-28.
19. Ramadan M, Tawfik S, El-Danasoury I, et al. Development of an Arabic remediation program for central auditory processing disorder in children, In: International Association of Logopedics and Phoniatrics: Proceedings CAPD Composium, Cairo, Egypt. 2009.

20. Tawfik S, Hazzaa N, Shalaby A, et al. Development of an Arabic battery for remediation of selective auditory attention disorder in children. Scand Audiol Suppl. 2001;52:211-216.

21. Tawfik S, El Danasoury I, Shalaby A, et al. Computer-based remediation program for auditory temporal processing disorders. Scientific Journal of Al-Azhar Medical Faculty Girls. 2007 ;28(2): 537-551.

22. Tawfik S, Shalaby A, El-Kholy W, et al. Development of Arabic Computer Based Remediation Program for Children with Central Auditory deficits. Presented in IFOS Conference. 2002.

23. Tawfik S, Shalaby S, El Kholi W, et al. Development of an Arabic computer-based remediation program for children with central auditory processing disorders. Presented in $28^{\text {th }}$ International Congress of Audiology-Innsbruck, Austria. 2006.

24. Tawfik S, Hassan D, Mesallamy R. Evaluation of long term outcome of auditory training programs in children with auditory processing disorders. ol. Int J Pediatr Otorhinolaryng. 2015; 79(12):2404-2410.

25. Afifi P, Kamal N, Tawfik S, et al. Efficacy of Auditory Processing Training in Cochlear Implant Children: A New Approach. Unpublished doctor thesis, Ain Shams University, Egypt. 2009. 